\title{
Control and Synchronization of the Fractional-Order Lorenz Chaotic System via Fractional-Order Derivative
}

\author{
Ping Zhou, ${ }^{1,2}$ and Rui Ding1, \\ ${ }^{1}$ Center of System Theory and Its Applications, Chongqing University of Posts and Telecommunications, \\ Chongqing 400065, China \\ ${ }^{2}$ Key Laboratory of Industrial Internet of Things \& Networked Control, Chongqing University of Posts and \\ Telecommunications, Ministry of Education, Chongqing 400065, China
}

Correspondence should be addressed to Ping Zhou, zhouping@cqupt.edu.cn

Received 8 February 2012; Revised 6 April 2012; Accepted 20 April 2012

Academic Editor: Ricardo Femat

Copyright (C) 2012 P. Zhou and R. Ding. This is an open access article distributed under the Creative Commons Attribution License, which permits unrestricted use, distribution, and reproduction in any medium, provided the original work is properly cited.

The unstable equilibrium points of the fractional-order Lorenz chaotic system can be controlled via fractional-order derivative, and chaos synchronization for the fractional-order Lorenz chaotic system can be achieved via fractional-order derivative. The control and synchronization technique, based on stability theory of fractional-order systems, is simple and theoretically rigorous. The numerical simulations demonstrate the validity and feasibility of the proposed method.

\section{Introduction}

The theory of fractional-order derivatives can be dated back to the 17th century [1] and developed comprehensively in the last century due to its application in a wide variety of scientific and technological fields such as thermal, viscoelastic, acoustic, electrochemical, rheological, and polymeric disciplines [1,2]. On the other hand, it has been shown that many fractional-order dynamical systems, as some well-known integer-order systems, can also display complex bifurcation and chaotic phenomena. For example, the fractional-order Lorenz system, the fractional-order Chen system, the fractional-order Lü system, and the fractional-order unified system also exhibit chaotic behavior. Due to its potential applications in secure communication and control processing, the fractional-order chaotic systems have been studied extensively in recent years in many aspects such as chaotic phenomena, chaotic control, chaotic synchronization, and other related studies [3-12].

It is verified that the fractional-order controllers can have better disturbance rejection ratios and less sensitivity to plant parameter variations compared to the traditional 
controllers [13]. A fractional-order controller is presented to stabilize the unstable equilibrium points of integer orders chaos systems $[13,14]$. But the previously presented in $[13,14]$ only discussed the control problem for integer orders chaos systems, not for fractionalorder chaotic systems. Up to now, to the best of our knowledge, amongst all kinds of chaos control and chaos synchronization for the fractional-order chaotic systems, very few results on control and synchronization of fractional-order chaotic systems are presented via fractional-order derivative. Motivated by the above discussion, a novel control method for the fractional-order Lorenz chaotic system is investigated in this paper. A fractionalorder controller is presented to stabilize the unstable equilibrium points of the fractionalorder Lorenz chaotic system via fractional-order derivative, and a fractional-order controller is presented to synchronize the fractional-order Lorenz chaotic system via fractional-order derivative. The control and synchronization technique, based on stability theory of fractionalorder systems, is simple and theoretically rigorous. The numerical simulations demonstrate the validity and feasibility of the proposed method.

\section{The Fractional Derivatives and the Fractional-Order Lorenz Chaotic System}

The Caputo definition of the fractional derivative, which sometimes is called smooth fractional derivative, is described as

$$
D^{q} f(t)=\frac{1}{\Gamma(m-q)} \int_{0}^{t} \frac{f^{(m)}(\tau)}{(t-\tau)^{q+1-m}} d \tau, \quad m-1<q<m,
$$

where $D^{q}$ denotes the Caputo definition of the fractional derivative. $m$ is the smallest integer larger than $q$, and $f^{(m)}(t)$ is the $m$-order derivative in the usual sense. $\Gamma(\cdot)$ is gamma function.

The Lorenz system, as the first chaotic model, revealed the complex and fundamental behaviors of the nonlinear dynamical systems. In 2003, I. Grigorenko and E. Grigorenko [4] pointed out that the fractional-order Lorenz system exhibits chaotic behavior for fractionalorder $q \geq 0.993$. The fractional-order Lorenz system is described as follows:

$$
\begin{gathered}
D^{q} x_{1}=10\left(x_{2}-x_{1}\right), \\
D^{q} x_{2}=28 x_{1}-x_{2}-x_{1} x_{3}, \\
D^{q} x_{3}=x_{1} x_{2}-\frac{8 x_{3}}{3},
\end{gathered}
$$

where $0<q<1$. In this paper, we choose $q=0.995$ for the fractional-order Lorenz chaotic system.

Now, we discuss the numerical solution of fractional differential equations. All the numerical simulation of fractional-order system in this paper is based on [3]. We can set 
$h=T / N, t_{n}=n h(n=0,1,2 \ldots, N)$, and initial condition $\left(x_{1}(0), x_{2}(0), x_{3}(0)\right)$. So, the fractional-order Lorenz chaotic system (2.2) can be discretized as follows:

$$
\begin{aligned}
x_{1}(n+1)=x_{1}(0)+\frac{h^{q}}{\Gamma(q+2)}[ & {\left[10\left(x_{2}^{p}(n+1)-x_{1}^{p}(n+1)\right)+\sum_{j=0}^{n} \alpha_{1, j, n+1} \times 10\left(x_{2}(j)-x_{1}(j)\right)\right], } \\
x_{2}(n+1)=x_{2}(0)+\frac{h^{q}}{\Gamma(q+2)}[ & {\left[28 x_{1}^{p}(n+1)-x_{2}^{p}(n+1)-x_{1}^{p}(n+1) x_{3}^{p}(n+1)\right.} \\
& \left.+\sum_{j=0}^{n} \alpha_{2, j, n+1}\left(28 x_{1}(j)-x_{2}(j)-x_{1}(j) x_{3}(j)\right)\right], \\
x_{3}(n+1)=x_{3}(0)+\frac{h^{q}}{\Gamma(q+2)}\left[\begin{array}{c}
x_{1}^{p}(n+1) x_{2}^{p}(n+1)-\frac{8 x_{3}^{p}(n+1)}{3} \\
+
\end{array}\right. & \left.\sum_{j=0}^{n} \alpha_{3, j, n+1}\left(x_{1}(j) x_{2}(j)-\frac{8 x_{3}(j)}{3}\right)\right],
\end{aligned}
$$

where

$$
\begin{gathered}
x_{1}^{p}(n+1)=x_{1}(0)+\frac{1}{\Gamma(q)} \sum_{j=0}^{n} b_{1, j, n+1} \times 10\left(x_{2}(j)-x_{1}(j)\right), \\
x_{2}^{p}(n+1)=x_{2}(0)+\frac{1}{\Gamma(q)} \sum_{j=0}^{n} b_{2, j, n+1}\left(28 x_{1}(j)-x_{2}(j)-x_{1}(j) x_{3}(j)\right), \\
x_{3}^{p}(n+1)=x_{3}(0)+\frac{1}{\Gamma(q)} \sum_{j=0}^{n} b_{3, j, n+1}\left(x_{1}(j) x_{2}(j)-\frac{8 x_{3}(j)}{3}\right),
\end{gathered}
$$

and for $i=1,2,3$,

$$
\begin{aligned}
& \alpha_{i, j, n+1}= \begin{cases}n^{q+1}-(n-q)(n+1)^{q}, & j=0, \\
(n-j+2)^{q+1}+(n-j)^{q+1}-2(n-j+1)^{q+1}, & 1 \leq j \leq n, \\
1, & j=n+1,\end{cases} \\
& b_{i, j, n+1}=\frac{h^{q}}{q}\left[(n-j+1)^{q}-(n-j)^{q}\right], \quad 0 \leq j \leq n .
\end{aligned}
$$

The error of this approximation is described as follows:

$$
\left|x_{i}\left(t_{n}\right)-x_{i}(n)\right|=o\left(h^{p}\right), \quad p=\min (2,1+q) .
$$




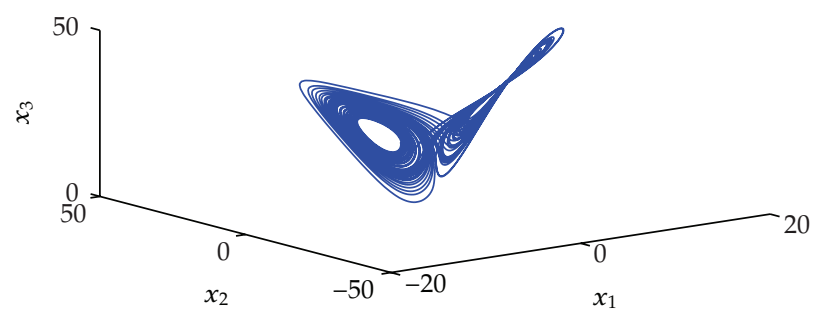

(a)

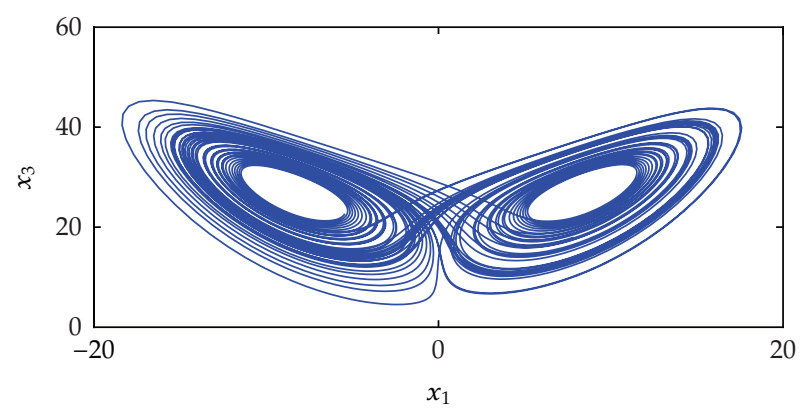

(b)

Figure 1: Chaotic attractors of the fractional-order Lorenz chaotic system (2.2) for $q=0.995$.

Using the above numerical solution for fractional-order Lorenz chaotic system (2.2), the chaotic attractor of fractional-order Lorenz chaotic system (2.2) for $q=0.995$ is shown in Figure 1.

\section{Stabilizing the Unstable Equilibrium Points of the Fractional-Order Lorenz Chaotic System via Fractional-Order Derivative}

It is obvious that the fractional-order Lorenz chaotic system (2.2) has three unstable equilibrium points. The unstable equilibrium points are $p_{0}=(0,0,0)$ and $p_{ \pm}=( \pm \sqrt{72}, \pm \sqrt{72}, 27)$. In this section, we will discuss how to stabilize the unstable equilibrium points of the fractional-order Lorenz chaotic system (2.2) via fractional-order derivative. First, let us present the stability theorem for linear commensurate fractional-order systems and nonlinear commensurate fractional-order systems.

Lemma 3.1 (see $[13,15])$. The following linear commensurate fractional-order autonomous system

$$
D^{q} x=A x, \quad x(0)=x_{0}
$$

is asymptotically stable if and only if $|\arg \lambda|>0.5 \pi q$ is satisfied for all eigenvalues $(\lambda)$ of matrix $A$. Also, this system is stable if and only if $|\arg \lambda| \geq 0.5 \pi q$ is satisfied for all eigenvalues of matrix $A$, and those critical eigenvalues which satisfy $|\arg \lambda|=0.5 \pi q$ have geometric multiplicity one, where $0<q<1, x \in R^{n}$ and $A \in R^{n \times n}$. 
Lemma 3.2 (see $[16,17])$. The fixed points of the following nonlinear commensurate fractional-order autonomous system:

$$
D^{q} x=f(x)
$$

is asymptotically stable if all eigenvalues ( $\lambda$ ) of the Jacobian matrix $A=\partial f / \partial x$ evaluated at the fixed points satisfy $|\arg \lambda|>0.5 \pi q$, where $0<q<1, x \in R^{n}, f: R^{n} \rightarrow R^{n}$ are continuous nonlinear vector functions, and the fixed points of this nonlinear commensurate fractional-order system are calculated by solving equation $f(x)=0$.

\subsection{Stabilizing the Unstable Equilibrium Point $p_{0}=(0,0,0)$ via Fractional-Order Derivative}

Now, let us design a controller for fractional-order Lorenz chaotic system (2.2) via fractionalorder derivative, and we can obtain the following results.

Theorem 3.3. Let the controlled fractional-order Lorenz chaotic system be

$$
\begin{gathered}
D^{q} x_{1}=10\left(x_{2}-x_{1}\right), \\
D^{q} x_{2}=28 x_{1}-x_{2}-x_{1} x_{3}+u_{1}\left(x_{1}\right), \\
D^{q} x_{3}=x_{1} x_{2}-\frac{8 x_{3}}{3}
\end{gathered}
$$

where $u_{1}\left(x_{1}\right)=-k_{11} D^{q} x_{1}-k_{12} x_{1}$ is the fractional-order controller, and $k_{1 i}(i=1,2)$ is the feedback coefficient. If $k_{12}=38+10 k_{11}$ and $-10 k_{11}-11 \leq 0$, then system (3.3) will asymptotically converge to the unstable equilibrium point $p_{0}=(0,0,0)$.

Proof. The Jacobi matrix of the controlled fractional-order Lorenz chaotic system (3.3) at equilibrium $p_{0}=(0,0,0)$ is

$$
J=\left[\begin{array}{ccc}
-10 & 10 & 0 \\
28+10 k_{11}-k_{12} & -10 k_{11}-1 & 0 \\
0 & 0 & -\frac{8}{3}
\end{array}\right]
$$

Because $k_{12}=38+10 k_{11}$, so

$$
J=\left[\begin{array}{ccc}
-10 & 10 & 0 \\
-10 & -10 k_{11}-1 & 0 \\
0 & 0 & -\frac{8}{3}
\end{array}\right]
$$




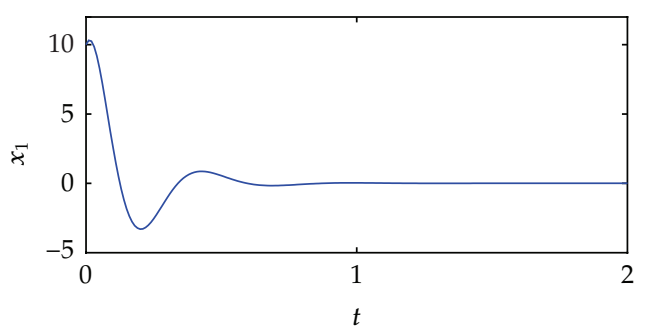

(a)

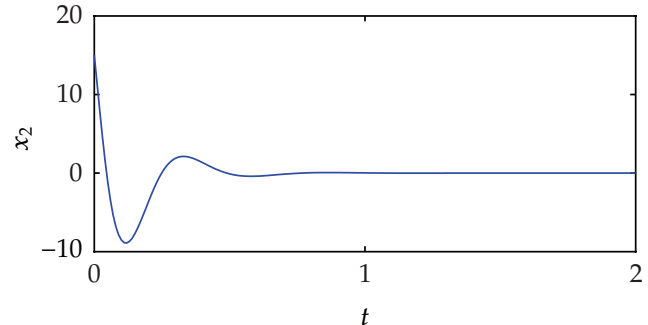

(b)

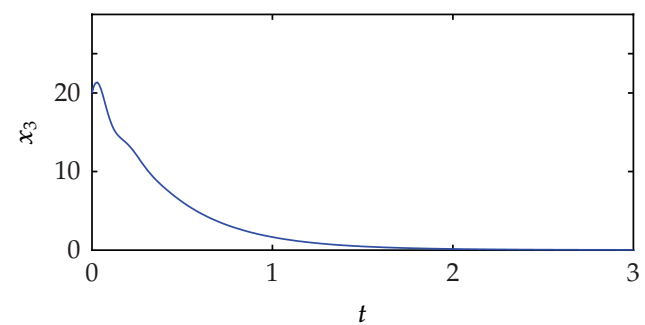

(c)

Figure 2: Time evolution of the equilibrium point $p_{0}=(0,0,0)$.

Its characteristic equation is $(\lambda+8 / 3)\left[\lambda^{2}+\left(10 k_{11}+11\right) \lambda+10\left(10 k_{11}+1\right)+100\right]=0$, and the eigenvalues are

$$
\lambda_{1,2}=\frac{-\left(10 k_{11}+11\right) \pm \sqrt{\left(10 k_{11}+11\right)^{2}-40\left(10 k_{11}+11\right)}}{2}, \quad \lambda_{3}=-\frac{8}{3}
$$

Because $-10 k_{11}-11 \leq 0$, so

$$
\left|\arg \lambda_{i}\right|>\frac{\pi}{2}>0.5 \pi q, \quad(i=1,2,3) .
$$

According to Lemma 3.2, it implies that the equilibrium point $p_{0}=(0,0,0)$ of system (3.3) is asymptotically stable, that is, the unstable equilibrium point $p_{0}=(0,0,0)$ in fractionalorder Lorenz system (2.2) can be stabilized via fractional-order derivative. The proof is completed.

For example, choose $k_{11}=0.1$, then $k_{12}=39$. The corresponding numerical result is shown in Figure 2, in which the initial conditions are $(10,15,20)^{T}$ in this paper.

Theorem 3.4. Consider the controlled fractional-order Lorenz chaotic system as follows:

$$
\begin{gathered}
D^{q} x_{1}=10\left(x_{2}-x_{1}\right)+u_{2}\left(x_{2}\right), \\
D^{q} x_{2}=28 x_{1}-x_{2}-x_{1} x_{3}, \\
D^{q} x_{3}=x_{1} x_{2}-\frac{8 x_{3}}{3}
\end{gathered}
$$


where $u_{2}\left(x_{2}\right)=-k_{21} D^{q} x_{2}-k_{22} x_{2}$ is the fractional-order controller, and $k_{2 i}(i=1,2)$ is the feedback coefficient. If $k_{22}=38-k_{21}$ and $-28 k_{21}-11 \leq 0$, then system (3.8) will gradually converge to the unstable equilibrium point $p_{0}=(0,0,0)$.

Proof. The Jacobi matrix of the controlled fractional-order Lorenz chaotic system (3.8) at equilibrium $p_{0}=(0,0,0)$ is

$$
J=\left[\begin{array}{ccc}
-10-28 k_{21} & 10-k_{21}-k_{22} & 0 \\
28 & -1 & 0 \\
0 & 0 & -\frac{8}{3}
\end{array}\right] .
$$

If $k_{22}=38-k_{21}$, then the Jacobi matrix is

$$
J=\left[\begin{array}{ccc}
-10-28 k_{21} & -28 & 0 \\
28 & -1 & 0 \\
0 & 0 & -\frac{8}{3}
\end{array}\right] .
$$

Its characteristic equation is $(\lambda+8 / 3)\left[\lambda^{2}+\left(28 k_{21}+11\right) \lambda+\left(28 k_{21}+10\right)+784\right]=0$, and the eigenvalues are

$$
\lambda_{1,2}=\frac{-\left(28 k_{21}+11\right) \pm \sqrt{\left(28 k_{21}+11\right)^{2}-4\left[\left(28 k_{21}+10\right)+784\right]}}{2}, \quad \lambda_{3}=-\frac{8}{3} .
$$

Because $-28 k_{21}-11 \leq 0$, so

$$
\left|\arg \lambda_{i}\right|>\frac{\pi}{2}>0.5 \pi q, \quad(i=1,2,3) .
$$

According to Lemma 3.2, it implies that the equilibrium point $p_{0}=(0,0,0)$ of system (3.8) is asymptotically stable, that is, the unstable equilibrium point $p_{0}=(0,0,0)$ in fractionalorder Lorenz system (2.2) can be stabilized via fractional-order derivative. The proof is completed.

For example, choose $k_{21}=0.1$, then $k_{22}=37.9$. The corresponding numerical result is shown in Figure 3.

\subsection{Stabilizing the Unstable Equilibrium Point $p_{ \pm}=( \pm \sqrt{72}, \pm \sqrt{72}, 27)$ via Fractional-Order Derivative}

Let us design a fractional-order controller for fractional-order Lorenz chaotic system (2.2), and we can yield the following results. 


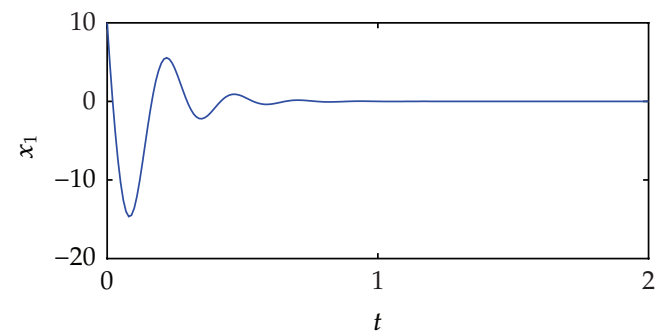

(a)

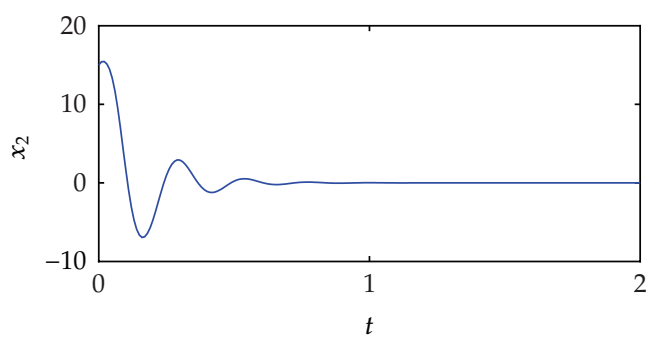

(b)

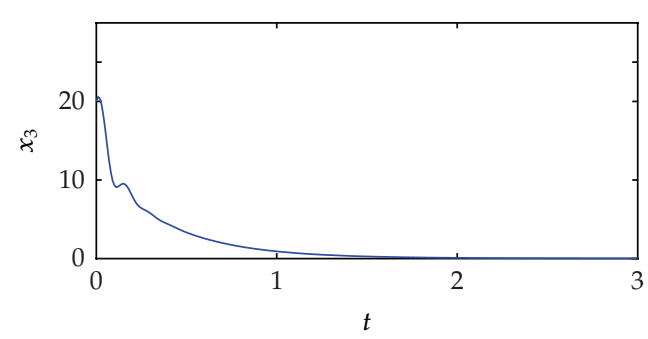

(c)

Figure 3: Time evolution of the equilibrium point $p_{0}=(0,0,0)$.

Theorem 3.5. Consider that the controlled fractional-order Lorenz chaotic system is

$$
\begin{gathered}
D^{q} x_{1}=10\left(x_{2}-x_{1}\right) \\
D^{q} x_{2}=28 x_{1}-x_{2}-x_{1} x_{3}+w_{1}\left(x_{1}\right) \\
D^{q} x_{3}=x_{1} x_{2}-\frac{8 x_{3}}{3}
\end{gathered}
$$

where $w_{1}\left(x_{1}\right)=-k_{1} D^{q} x_{1}$ is the fractional-order controller, and $k_{1}$ is the feedback coefficient. If $k_{1}>$ $(-155+\sqrt{24769}) / 60$, then system (3.13) will gradually converge to the unstable equilibrium point $p_{+}=(\sqrt{72}, \sqrt{72}, 27)$.

Proof. Let $x=x_{1}-\sqrt{72}, y=x_{2}-\sqrt{72}, z=x_{3}-27$, and the controlled fractional-order Lorenz chaotic system (3.13) can be

$$
\begin{gathered}
D^{q} x=10(y-x), \\
D^{q} y=x-y-x z-\sqrt{72} z-k_{1} D^{q} x \\
D^{q} z=x y+\sqrt{72} x+\sqrt{72} y-\frac{8 z}{3} .
\end{gathered}
$$




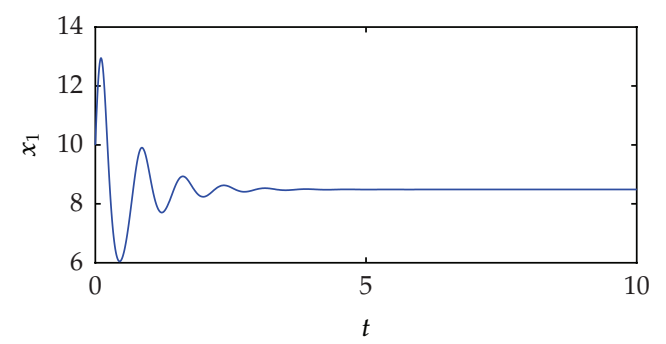

(a)

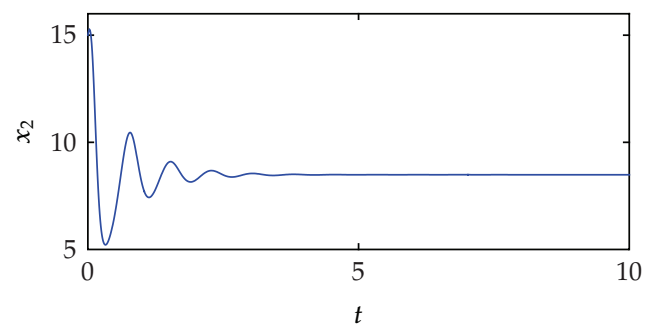

(b)

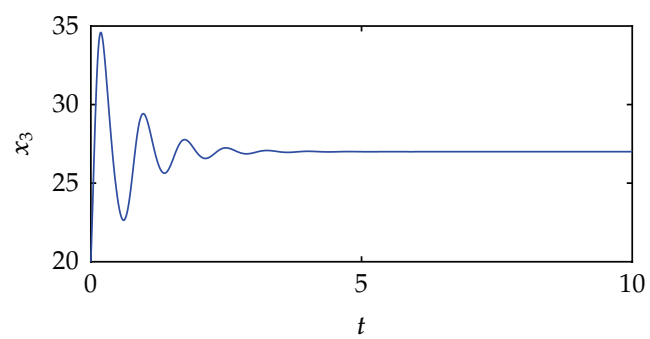

(c)

Figure 4: Time evolution of the equilibrium point $p_{+}=(\sqrt{72}, \sqrt{72}, 27)$.

The Jacobi matrix of the fractional-order system (3.14) at equilibrium $(0,0,0)$ is

$$
J=\left[\begin{array}{ccc}
-10 & 10 & 0 \\
10 k_{1}+1 & -10 k_{1}-1 & -\sqrt{72} \\
\sqrt{72} & \sqrt{72} & -\frac{8}{3}
\end{array}\right] .
$$

Its characteristic equation is $\lambda^{3}+a_{1} \lambda^{2}+a_{2} \lambda+a_{3}=0$, where $a_{1}=10 k_{1}+41 / 3, a_{2}=$ $8\left(10 k_{1}+11\right) / 3+72$, and $a_{3}=1440$. If $k_{1}>(-155+\sqrt{24769}) / 60$, then $a_{1}>0, a_{2}>0, a_{3}>0$, and $a_{1} a_{2}-a_{3}>0$. So,

$$
\left|\arg \lambda_{i}\right|>\frac{\pi}{2}>0.5 \pi q, \quad(i=1,2,3) .
$$

According to Lemma 3.2, it implies that the equilibrium point $p_{+}=(\sqrt{72}, \sqrt{72}, 27)$ of system (3.13) is asymptotically stable, that is, the unstable equilibrium point $p_{+}=(\sqrt{72}$, $\sqrt{72}, 27)$ in fractional-order Lorenz system $(2.2)$ can be stabilized via fractional-order derivative. The proof is completed.

For example, choose $k_{1}=1$. The corresponding numerical result is shown in Figure 4 . 
Theorem 3.6. Consider that the controlled fractional-order Lorenz chaotic system is

$$
\begin{gathered}
D^{q} x_{1}=10\left(x_{2}-x_{1}\right)+w_{2}\left(x_{2}\right), \\
D^{q} x_{2}=28 x_{1}-x_{2}-x_{1} x_{3}, \\
D^{q} x_{3}=x_{1} x_{2}-\frac{8 x_{3}}{3},
\end{gathered}
$$

where $w_{2}\left(x_{2}\right)=-k_{2} D^{q} x_{2}$ is the fractional-order controller, and $k_{2}$ is the feedback coefficient. If $(-7616-\sqrt{56765440}) / 1248<k_{2}<(-7616+\sqrt{56765440}) / 1248$, then system (3.17) will gradually converge to the unstable equilibrium point $p_{+}=(\sqrt{72}, \sqrt{72}, 27)$.

Proof. Let $x=x_{1}-\sqrt{72}, y=x_{2}-\sqrt{72}, z=x_{3}-27$, and the controlled fractional-order Lorenz chaotic system (3.17) can be

$$
\begin{gathered}
D^{q} x=10(y-x)-k_{2} D^{q} y, \\
D^{q} y=x-y-x z-\sqrt{72} z, \\
D^{q} z=x y+\sqrt{72} x+\sqrt{72} y-\frac{8 z}{3} .
\end{gathered}
$$

The Jacobi matrix of the fractional-order system (3.18) at equilibrium $(0,0,0)$ is

$$
J=\left[\begin{array}{ccc}
-10-k_{2} & 10+k_{2} & k_{2} \sqrt{72} \\
1 & -1 & -\sqrt{72} \\
\sqrt{72} & \sqrt{72} & -\frac{8}{3}
\end{array}\right] .
$$

Its characteristic equation is $\lambda^{3}+a_{1} \lambda^{2}+a_{2} \lambda+a_{3}=0$, where $a_{1}=k_{2}+41 / 3, a_{2}=$ $8\left(k_{2}+11\right) / 3+72\left(1-k_{2}\right)$, and $a_{3}=1440$. If $(-7616-\sqrt{56765440}) / 1248<k_{2}<(-7616+$ $\sqrt{56765440}) / 1248$, then $a_{1}>0, a_{2}>0, a_{3}>0$, and $a_{1} a_{2}-a_{3}>0$. So,

$$
\left|\arg \lambda_{i}\right|>\frac{\pi}{2}>0.5 \pi q, \quad(i=1,2,3)
$$

According to Lemma 3.2, it implies that the equilibrium point $p_{+}=(\sqrt{72}, \sqrt{72}, 27)$ of system (3.17) is asymptotically stable, that is, the unstable equilibrium point $p_{+}=(\sqrt{72}$, $\sqrt{72}, 27)$ in fractional-order Lorenz system $(2.2)$ can be stabilized via fractional-order derivative. The proof is completed.

For example, choose $k_{2}=-2$. The corresponding numerical result is shown in Figure 5 .

Similarly, the fractional-order Lorenz chaotic system can be easily controlled to another unstable equilibrium point $p_{-}=(-\sqrt{72},-\sqrt{72}, 27)$. 


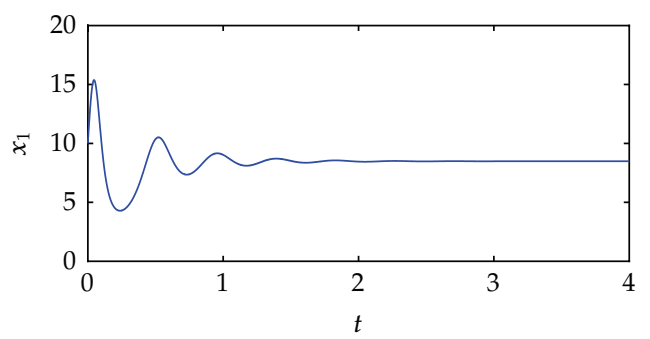

(a)

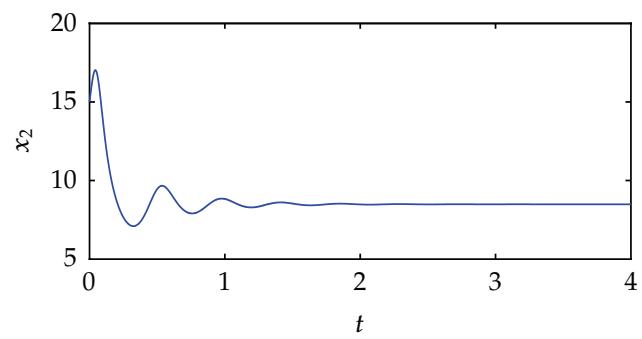

(b)

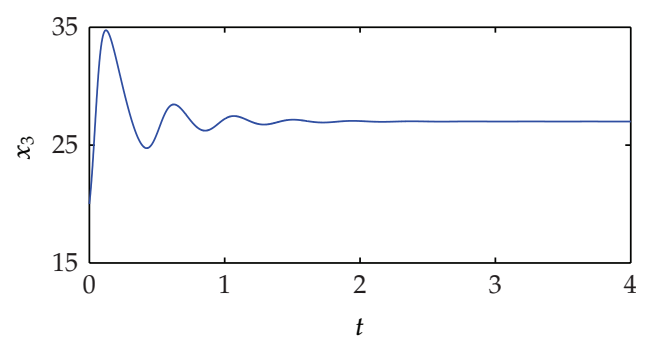

(c)

Figure 5: Time evolution of the equilibrium point $p_{+}=(\sqrt{72}, \sqrt{72}, 27)$.

Remark 3.7. In general, there is no universal method to select the controller, and these particular controllers in our paper depend on the structure of the fractional-order chaotic system.

Remark 3.8. The differences of the present control strategy in our paper are compared to the result reported by Tavazoei and Haeri [13] as follows. First, we use the scalar controller in our paper, but they used the vector controller. Second, we discuss the control problem for fractional-order chaotic systems via fractional-order derivative, but they discussed the control problem for integer orders chaos systems via fractional-order derivative.

Remark 3.9. The differences of the present control strategy in our paper are compared to the result reported by Razminia et al. [18] as follows. We discuss the control problem for fractional-order chaotic systems via fractional-order derivative, but they discussed the control problem for fractional-order chaotic systems via state feedback, and they did not use fractional-order derivative.

\section{Synchronizing the Fractional-Order Lorenz Chaotic System via Fractional-Order Derivative}

Now, we design a feedback controller for the fractional-order Lorenz chaotic system (2.2) via fractional-order derivative and obtain the controlled response system (4.1)

$$
\begin{gathered}
D^{q} y_{1}=10\left(y_{2}-y_{1}\right), \\
D^{q} y_{2}=28 y_{1}-y_{2}-y_{1} y_{3}+V, \\
D^{q} y_{3}=y_{1} y_{2}-\frac{8 y_{3}}{3},
\end{gathered}
$$


where $V=k_{1}\left(D^{q} y_{1}-D^{q} x_{1}\right)+k_{2}\left(y_{1}-x_{1}\right)+y_{1} y_{3}-x_{1} x_{3}$ is the fractional-order controller, and $k_{i}(i=1,2)$ is the feedback coefficient. Now, we can yield the following theorem.

Theorem 4.1. If the feedback coefficients $k_{i}(i=1,2)$ satisfy

$$
28-10 k_{1}+k_{2}=-10, \quad-1+10\left(k_{1}-1\right)<0,
$$

then the fractional-order Lorenz chaotic system (2.2) and the controlled fractional-order Lorenz chaotic system (4.1) achieved synchronization via fractional-order derivative.

Proof. Define the synchronization error variables as follows:

$$
e_{i}=y_{i}-x_{i}, \quad(i=1,2,3)
$$

By subtracting (2.2) from (4.1), we obtain

$$
\left(\begin{array}{c}
D^{q} e_{1} \\
D^{q} e_{2} \\
D^{q} e_{3}
\end{array}\right)=A\left(\begin{array}{l}
e_{1} \\
e_{2} \\
e_{3}
\end{array}\right)
$$

where

$$
A=\left[\begin{array}{ccc}
-10 & 10 & 0 \\
28-10 k_{1}+k_{2} & -1+10 k_{1} & 0 \\
x_{2} & y_{1} & -\frac{8}{3}
\end{array}\right] \text {. }
$$

Because $28-10 k_{1}+k_{2}=-10$, so

$$
A=\left[\begin{array}{ccc}
-10 & 10 & 0 \\
-10 & -1+10 k_{1} & 0 \\
x_{2} & y_{1} & -\frac{8}{3}
\end{array}\right]
$$

Therefore, the eigenvalues are

$$
\lambda_{1,2}=\frac{\beta \pm \sqrt{\beta^{2}+40 \beta}}{2}, \quad \lambda_{3}=-\frac{8}{3}<0,
$$

where $\beta=-1+10\left(k_{1}-1\right)$.

Because $\beta=-1+10\left(k_{1}-1\right)<0$, then all the eigenvalues of matrix $A$ have negative real part. Therefore,

$$
\left|\arg \lambda_{i}(A)\right|>\frac{\pi}{2}>0.5 \pi q, \quad(i=1,2,3) .
$$




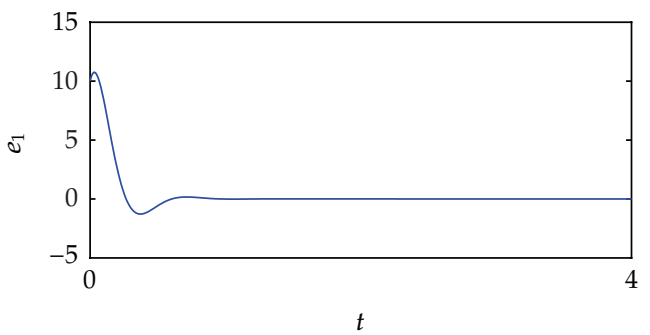

(a)

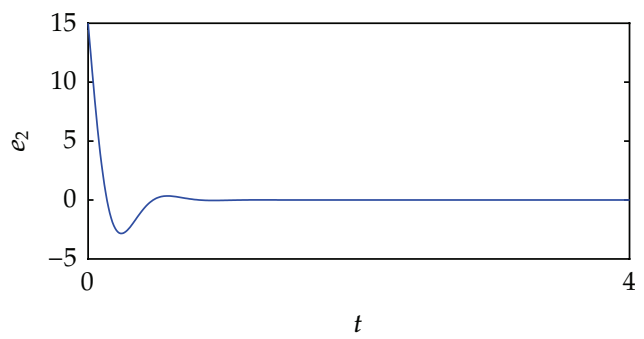

(b)

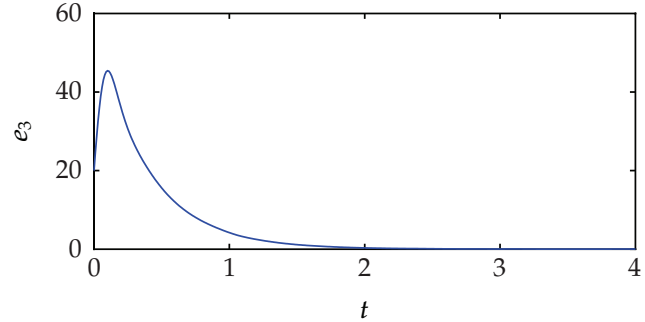

(c)

Figure 6: The time variation of synchronization error.

According to Lemma 3.2, it implies that the equilibrium point $(0,0,0)$ of error system (4.4) is asymptotically stable, that is, $\lim _{t \rightarrow+\infty} e_{i}=0(i=1,2,3)$. So, the fractional-order Lorenz chaotic system (2.2) and the controlled fractional-order Lorenz chaotic system (4.1) achieved synchronization via fractional-order derivative. The proof is completed.

Next, in order to verify the effectiveness and feasibility of the proposed synchronization scheme, the corresponding numerical simulations are given. For example, choose $k_{1}=-0.1$ and $k_{2}=-39$; the time variation of synchronization error is shown in Figure 6 . The initial conditions are $\mathbf{x}(0)=(10,20,30)^{\mathrm{T}}$, and $\mathbf{y}(0)=(20,35,50)^{\mathrm{T}}$ respectively.

\section{Conclusion}

Using fractional-order derivative, we can stabilize the unstable equilibrium points of the fractional-order Lorenz chaotic system and realize chaos synchronization for the fractionalorder Lorenz chaotic system. The control technique in our paper is simple and theoretically rigorous. Some examples are also given to illustrate the effectiveness of the theoretical result. This proposed control method is different from the previous works and can be applied to other fractional-order chaotic systems.

\section{Acknowledgments}

The authors are very grateful to the reviewers for their valuable comments and suggestions. This work was supported by the Foundation of Science and Technology project of Chongqing Education Commission (KJ110525) and by the National Natural Science Foundation of China (61104150). 


\section{References}

[1] I. Podlubny, Fractional Differential Equations, Academic Press, New York, NY, USA, 1999.

[2] R. Hilfer, Applications of Fractional Calculus in Physics, World Scientific, New Jersey, NJ, USA, 2001.

[3] C. Li and G. Peng, "Chaos in Chen's system with a fractional order," Chaos, Solitons and Fractals, vol. 22, no. 2, pp. 443-450, 2004.

[4] I. Grigorenko and E. Grigorenko, "Chaotic dynamics of the fractional Lorenz system," Physical Review Letters, vol. 91, no. 3, Article ID 034101, 4 pages, 2003.

[5] X. Y. Wang and J. M. Song, "Synchronization of the fractional order hyperchaos Lorenz systems with activation feedback control," Communications in Nonlinear Science and Numerical Simulation, vol. 14, no. 8, pp. 3351-3357, 2009.

[6] G. H. Erjaee and M. Alnasr, "Phase synchronization in coupled Sprott chaotic systems presented by fractional differential equations," Discrete Dynamics in Nature and Society, vol. 2009, Article ID 753746, 10 pages, 2009.

[7] H. Taghvafard and G. H. Erjaee, "Phase and anti-phase synchronization of fractional order chaotic systems via active control," Communications in Nonlinear Science and Numerical Simulation, vol. 16, no. 10, pp. 4079-4088, 2011.

[8] Y. Yu, H. X. Li, S. Wang, and J. Yu, "Dynamic analysis of a fractional-order Lorenz chaotic system," Chaos, Solitons and Fractals, vol. 42, no. 2, pp. 1181-1189, 2009.

[9] S. Yang, J. Wu, M. Li, and A. Xiao, "Generations and mechanisms of multi-stripe chaotic attractors of fractional order dynamic system," Applied Mathematics and Computation, vol. 217, no. 13, pp. 62196229, 2011.

[10] L. Chen, Y. Chai, and R. Wu, "Lag projective synchronization in fractional-order chaotic (hyperchaotic) systems," Physics Letters A, vol. 375, no. 21, pp. 2099-2110, 2011.

[11] B. Xin, T. Chen, and Y. Liu, "Projective synchronization of chaotic fractional-order energy resources demand-supply systems via linear control," Communications in Nonlinear Science and Numerical Simulation, vol. 16, no. 11, pp. 4479-4486, 2011.

[12] S. Wang, Y. Yu, and M. Diao, "Hybrid projective synchronization of chaotic fractional order systems with different dimensions," Physica A, vol. 389, no. 21, pp. 4981-4988, 2010.

[13] M. S. Tavazoei and M. Haeri, "Chaos control via a simple fractional-order controller," Physics Letters A, vol. 372, no. 6, pp. 798-807, 2008.

[14] P. Zhou and F. Kuang, "A novel control method for integer orders chaos systems via fractional-order derivative," Discrete Dynamics in Nature and Society, vol. 2011, Article ID 217843, 2011.

[15] D. Matignon, "Stability result on fractional differential equations with applications to control processing," in Proceedings of the (IMACS-SMC '96), vol. 2, pp. 963-968, Lille, France, July 1996.

[16] K. Diethelm and N. J. Ford, "Analysis of fractional differential equations," Journal of Mathematical Analysis and Applications, vol. 265, no. 2, pp. 229-248, 2002.

[17] E. Ahmed, A. M. A. El-Sayed, and H. A. A. El-Saka, "Equilibrium points, stability and numerical solutions of fractional-order predator-prey and rabies models," Journal of Mathematical Analysis and Applications, vol. 325, no. 1, pp. 542-553, 2007.

[18] A. Razminia, V. J. Majd, and D. Baleanu, "Chaotic incommensurate fractional order Rössler system: active control and synchronization," Advances in Difference Equations, vol. 2011, no. 1, article 15, 2011. 


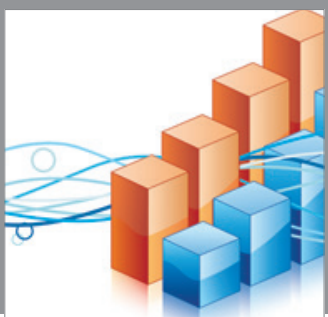

Advances in

Operations Research

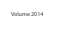

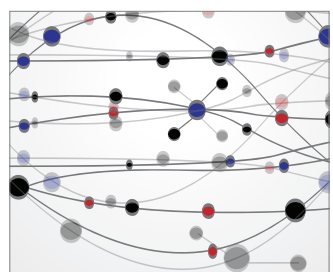

\section{The Scientific} World Journal
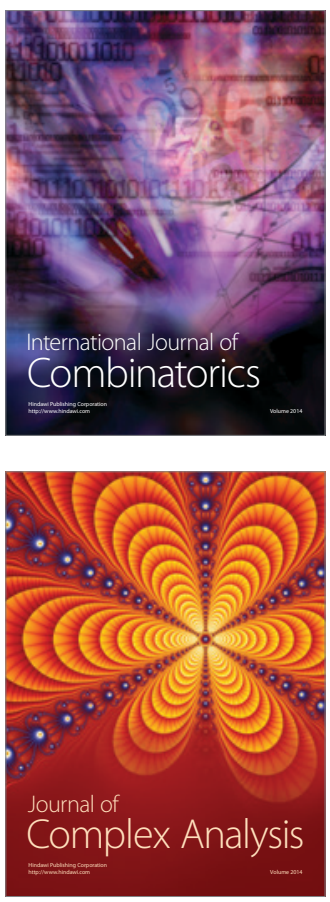

International Journal of

Mathematics and

Mathematical

Sciences
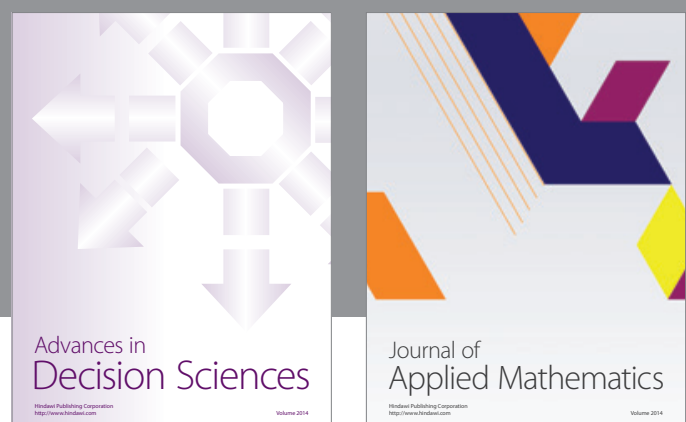

Journal of

Applied Mathematics
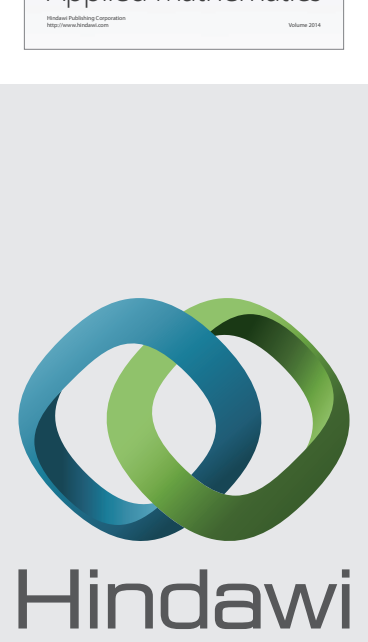

Submit your manuscripts at http://www.hindawi.com
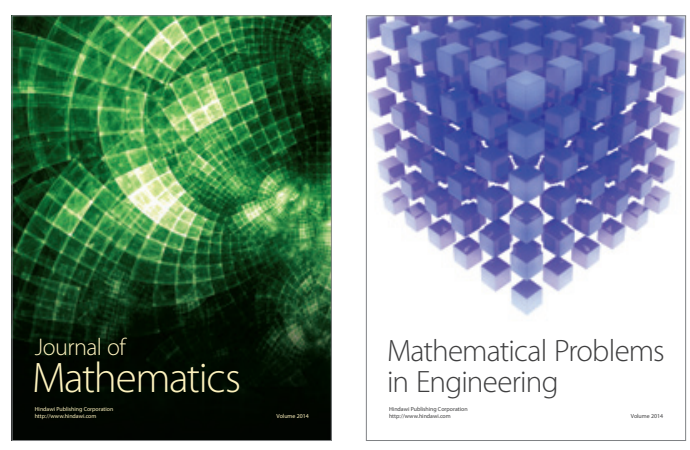

Mathematical Problems in Engineering
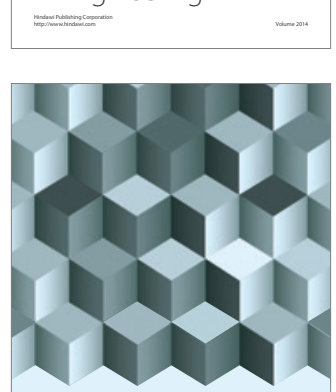

Journal of

Function Spaces
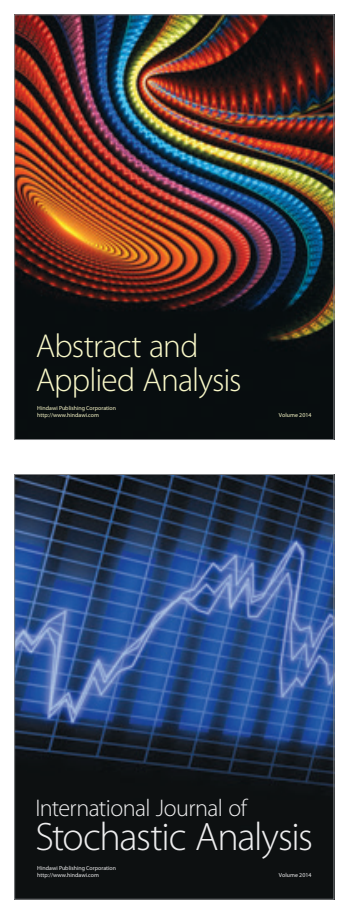

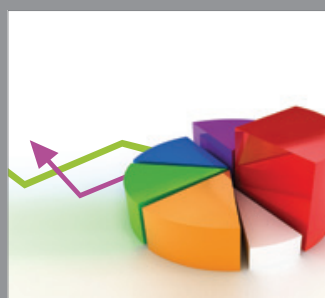

ournal of

Probability and Statistics

Promensencen
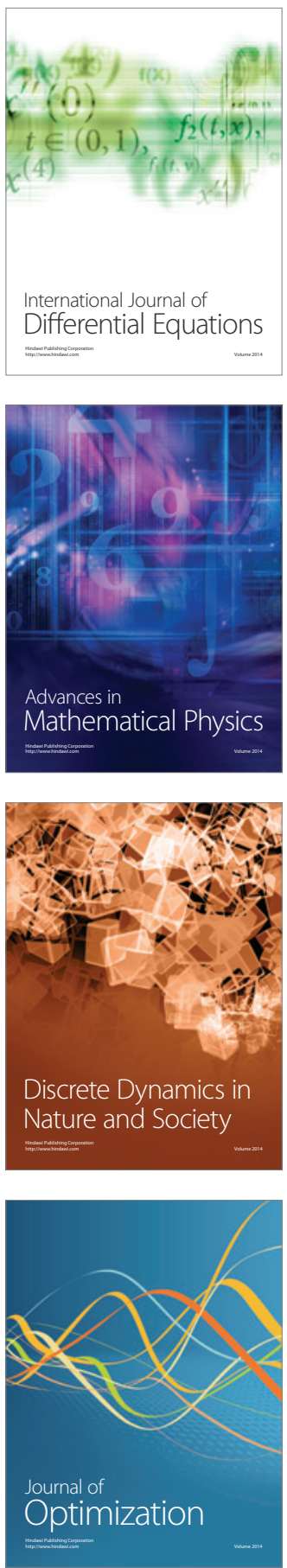